\title{
Nanoscale
}

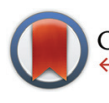

CrossMark \& click for updates

Cite this: Nanoscale, 2015, 7, 1136

Received 8th September 2014, Accepted 15th November 2014

DOI: $10.1039 / c 4 n r 05208 k$

www.rsc.org/nanoscale

\section{The real $\mathrm{TiO}_{2} / \mathrm{HTM}$ interface of solid-state dye solar cells: role of trapped states from a multiscale modelling perspective $\uparrow$} \author{
Agnese Abrusci, ${ }^{d}$ Henry J. Snaith, ${ }^{d}$ Giorgio Divitini, ${ }^{e}$ Caterina Ducati ${ }^{e}$ and
}

Alessio Gagliardi, ${ }^{\text {a }}$ Matthias Auf der Maur, ${ }^{\mathrm{b}}$ Desiree Gentilini, ${ }^{\mathrm{b}}$ Fabio di Fonzo, ${ }^{\mathrm{c}}$ Aldo Di Carlob

In this paper we present a multiscale simulation of charge transport in a solid-state dye-sensitized solar cell, where the real morphology between $\mathrm{TiO}_{2}$ and the hole transport material is included. The geometry of the interface is obtained from an electron tomography measurement and imported in a simulation software. Charge distribution, electric field and current densities are computed using the drift-diffusion model. We use this approach to investigate the electrostatic effect of trap states at the interface between the electron and hole transport materials. The simulations show that when the trapped electrons are not screened by external additives, the dynamics of holes is perturbed. Holes accumulate at the interface, enhancing recombination and reducing cell performance.
\end{abstract}

\section{Introduction}

Electronic technology has boomed in recent years mainly thanks to the enormous amount of research on materials science. A new class of materials is therefore emerging, i.e. organic semiconductors, which has opened interesting perspectives for electronic and optoelectronic devices. ${ }^{1-3}$ Organic semiconductors are becoming a crucial component for photovoltaic devices as well, where they can play either the role of active layer, as in organic photovoltaic cells (OPV), ${ }^{4}$ or hole transporter material, as in solid-state dye-sensitized solar cells (ss-DSCs). ${ }^{5}$ ss-DSCs have evolved from dye solar cells (DSCs), ${ }^{6,7}$ electrochemical photovoltaic devices that use a mesoporous oxide, usually titanium oxide $\left(\mathrm{TiO}_{2}\right)$, sensitized with a monolayer of molecules, the dye, to convert light into electricity. The $\mathrm{TiO}_{2}$ acts as an electron transport layer, while the dye is the active component that absorbs light. In standard DSCs, the role of hole transport layer is played by a liquid electrolyte containing a redox couple. However, several engineering problems

\footnotetext{
${ }^{a}$ Technische Universität München, Electrical and Computer Eng., Arcisstr. 21, 80333 München, Germany.E-mail: alessio.gagliardi@tum.de

${ }^{b}$ University of Rome "Tor Vergata", Via del Politecnico 1, 00133 Rome, Italy

${ }^{c}$ Center for Nano Science and Technology of IIT@PoliMI, Via Pascoli 70/3, 20133 Milano, Italy

${ }^{d}$ University of Oxford, Dep. of Physics, Clarendon Laboratory, Parks Road, Oxford OX1 3PU, UK

${ }^{e}$ University of Cambridge, Dep. of Materials Science and Metallurgy, 27 Charles Babbage road, Cambridge CB3 OFS, UK

$\dagger$ Electronic supplementary information (ESI) available. See DOI: 10.1039/ c4nr05208k
}

plague DSCs, such as the integrity of the liquid electrolyte. In fact, contamination from the external atmosphere or escaping of the electrolyte from microcracks within the sealant cause the efficiency of the cell to deteriorate over time, shortening the device's lifespan. Finding a good sealant for DSCs is still an open problem. ${ }^{8}$ For this reason the liquid hole transporter has been replaced with a solid one ${ }^{5}$ using both small molecule (such as 2,2',7,7'-Tetrakis-( $N, N$-di-4-methoxyphenylamino)9,9'-spirobifluorene (Spiro-OMeTAD)) or polymer (such as Poly(3-hexylthiophene-2,5-diyl) (P3HT)) organic semiconductors. ${ }^{9}$ Recently discovered perovskite solar cells have also emerged from DSC technology, starting from an effort to replace the dye ${ }^{10}$ and the hole transporter. ${ }^{11}$

The first prototypes of ss-DSCs had limited efficiency, but it was rapidly realized that the efficiency could be enhanced by doping the hole transport material with ionic additives such as lithium bis(trifluoromethylsulfonyl)-imide (Li-TFSI) in SpiroOMeTAD. ${ }^{12}$ The ionic additives are known to change many aspects of the bulk properties of the organic material as well as its interface characteristics. For example it is known that dopants change the density of holes, ${ }^{13}$ but also the holehopping mobility by smoothing the electrostatic landscape and easing the hopping process. ${ }^{14-16}$ They modify the optical properties of the dye, ${ }^{17,18}$ altering band alignment between $\mathrm{TiO}_{2}$ and the hole transport material by creating a dipole moment at the interface. ${ }^{19}$ Despite an intense investigation, many aspects of the doping are not completely clear in a working ss-DSC device, considering the complex interplay between many different processes affected by the dopants, which change the cell performance. For example, it has been 
demonstrated $^{13}$ that increasing the concentration of dopant Li-TFSI beyond $10 \%$ molarity does not further improve the efficiency of the cell, but still increases the fill factor (FF), suggesting that the dopants induce different effects that could also work in opposite directions.

The effect on the interface has been studied in detail and several aspects have been identified: one is the formation of a dipole moment, which shifts down the vacuum level of the $\mathrm{TiO}_{2}{ }^{19}$ the other is the effect of intercalation of lithium ions in the oxide, which induces an increase in electron trap states and a lower diffusivity of negative charges. ${ }^{20-22}$ Considering these last two effects, it would seem that adding ionic additives has mostly a bad influence on the cell. However, we should consider the determinant role of lithium ions in reducing the recombination. ${ }^{23}$ Several authors have pointed out that this is due to the presence of the positive lithium ions, which screen the electrostatic interaction between electrons and holes. ${ }^{24,25}$ Such screening is especially important between trapped electrons at the interface $\mathrm{e}^{26,27}$ and holes in the hole transport material.

The investigation of these effects is very complex considering that in experimental measurements, which usually consider the entire device, it is very hard to decouple the different physical/chemical mechanisms involved. Thus, theory and simulations can be helpful in trying to isolate different processes and evaluate their impact on the overall efficiency of the cell. Theoretical investigations on Ss-DSCs are generally focused on the coordination effect of these ionic additives ${ }^{28}$ and on the mobility of lithium ions. ${ }^{22}$ More recently, the effect of ionic additives on the hole conductivity of Spiro-OMeTAD has been assessed by means of a Kinetic Monte Carlo simulation. ${ }^{39}$ This allows to investigate from a more microscopic perspective the effect of additives on charge hopping by distinguishing between dopant additives (Li-TFSI, H-TFSI) and inactive additives, where no charge transfer occurs (Na-TFSI, Et4Na-TFSI). This study reveals the importance of electrostatic effects on hole conductivity induced by the introduction of an anion-cation pair in the organic matrix. Nevertheless, the gap between modelling of the whole device and experimental measurements is broad, and many questions are still open.

In particular, device modelling tends either to neglect nanoscale morphology by considering effective material properties or to assume highly idealized geometries. ${ }^{40}$ To assess the effect of the mesoscopic features of porous $\mathrm{TiO}_{2}$ in ss-DSC modeling, it is necessary to integrate information on the morphology into the simulation model. Such information can be obtained with high spatial resolution from electron tomography. ${ }^{41}$

In this work we present numerical simulations of charge transport in a working ss-DSC device, adopting an innovative multiscale approach to include the real interface between $\mathrm{TiO}_{2}$ and the hole transport material. We will focus on the electrostatic effect due to the interplay of ionic additives and electronic trap states at the interface. We consider two limiting cases. The first one concerns a cell without ionic additives, such that the electrostatics near the interface are governed by the charged traps. In the second case we assume a sufficiently high concentration of ionic additives, resulting in a complete screening of charged traps. In the latter case we consider only non-doping additives in order not to alter bulk properties, and we assume perfect screening with zero Debye length, such that the additives do not have to be included explicitly in the simulation model.

\section{Modelling}

Devices like DSCs pose a challenging problem for their modelling. The interface between the hole (HTM) and electron transport materials (ETM) is a fundamental part of the system, where most of the main processes (charge generation, recombination and trapping/detrapping events) occur. However, this interface is tortuous due to the nanoporous nature of the $\mathrm{TiO}_{2}$, and integration of the real interface in the simulation is very difficult, mainly due to two problems. The first concerns the difficulty in getting experimental measures of the actual geometry of such a complex structure. The second is related to the big difference in characteristic lengths between the mesoporous morphology (nm) and the thickness of the active layer of the device $(\mu \mathrm{m})$ : while the first is of the order of the diameter of the $\mathrm{TiO}_{2}$ nanoparticles used to form the mesoporous layer $(15 \mathrm{~nm})$, the second is in the range of a few micrometers, allowing the dye to absorb as much light as possible. The challenge is to handle the two different length scales within the same simulation. If in fact we describe the porous active layer with the resolution of the morphology, simulating the entire device is very computationally expensive since a large amount of information is needed to properly describe the exact interface geometry in the full volume of the solar cell. Commonly, this problem is overcome by using an effective material approximation. ${ }^{40}$ By effective material we mean that the real geometry of the interface is not included in the simulation. The electron and hole transport materials are treated as a single homogeneous material that fills the device. This effective material is parameterized using the ETM parameters for electrons and HTM parameters for holes. The main advantage of this approximation is that the complex structure of the interface is removed from the simulation model with a great simplification and reduction in computational cost. The characteristic length in this case reduces to the length of the active layer and thus it is in the range of micrometers. Moreover, it allows us to model the device even if the details of the morphology are unknown.

The main drawback is that all the processes (generation, recombination, etc.) that occur at the interface must be now converted into bulk models. Passing from interface to bulk models introduces approximation in the recombination and generation and their parameters should be adjusted to take into account the change in device description. Moreover, some aspects about the operation of the device, especially the charge distribution close to the interface, are definitely lost in this approximation.

In this work we use an intermediate approach: we describe part of the device using the resolution of the morphology, 


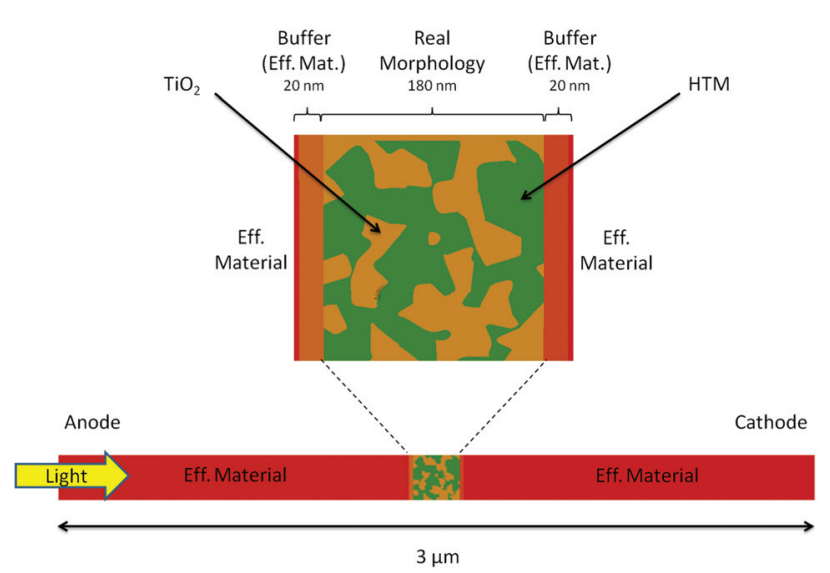

Fig. 1 Embedding of the real morphology (central region) within two regions modelled using the effective material for a $2 \mathrm{D}$ device. The mesh is divided in five parts, two regions close to the contacts (anode and cathode) with a less dense meshing, two buffering regions at the interface with a denser meshing but still using the effective material, and a central region with a 2D slice of the real blend. The entire device is $3 \mu \mathrm{m}$ long and the light comes from the left (anode) side. We assume periodic boundary conditions for the side boundaries of the cell.

while the rest of the active layer is simulated using an effective material approximation (see Fig. 1). This strategy allows us to model the entire system and thus to analyze the performance of the cell, being able at the same time to describe the real interface in at least one sub-part of the active layer.

The morphology region has been constructed by using the experimental measurement of the interface geometry of a $\mathrm{TiO}_{2} /$ Spiro-OMeTAD ss-DSC. The meshing of the full 3D structure is a challenging task and leads to the high computational cost of the transport simulations. Therefore, the geometry has been simplified considering a 2D slice of the real morphology $(180 \mathrm{~nm} \times 180 \mathrm{~nm})$ embedded in two regions using the effective material. This dimensional reduction is not a too restricitve approximation, as it can still address the problem of local charge distribution and the interplay between free and trapped charges at the interface.

The numerical simulations are based on the drift-diffusion (DD) equations and solved using the finite element method (FEM), using the TiberCAD simulation tool. ${ }^{29-31}$ The finite element approach is particularly suited since it allows us to easily discretize complex geometries. DSCs are generally simulated in a $1 \mathrm{D}$ domain ${ }^{32-35}$ solving the diffusion equation only, considering that, especially for standard DSCs with liquid electrolyte, the long-range electric field is screened. Several attempts to analyze the system, including for example the electrostatic potential or going beyond the 1D approximation, have been performed. ${ }^{36}$ Other studies have investigated a complete DSC including the electrolyte, studying $2 \mathrm{D}^{37}$ and $3 \mathrm{D}^{44-46}$ effects or innovative architectures. ${ }^{47}$

In DD the current is calculated by coupling the constitutive equations for charge transport with the continuity equation for charge conservation:

$$
\nabla\left(\mu n_{\mathrm{e}(\mathrm{h})} \nabla \phi_{\mathrm{e}(\mathrm{h})}\right)=G-R,
$$

where $\mu$ is the mobility of the material, $n_{\mathrm{e}(\mathrm{h})}$ the electron (hole) density and $\phi_{\mathrm{e}(\mathrm{h})}$ the corresponding electrochemical potential. Finally, $G$ and $R$ are the generation and recombination models respectively. The drift-diffusion equation shown in eqn (1) is general and relies on the generalized Einstein relation between mobility and diffusion coefficient. We present here the transport equation in this shape because the organic semiconductor does not satisfy the standard Einstein relation and thus the common split between drift and diffusion components is not correct. The transport equations must be coupled to the Poisson equation to solve the electrostatic potential in the device:

$$
\varepsilon \Delta \phi=-\rho,
$$

with $\varepsilon$ the dielectric constant, $\phi$ the electrostatic potential and $\rho$ the sum of all charged species in the system, electrons, holes and trapped charges or ionic species.

The system under investigation is a mix of $\mathrm{TiO}_{2}$ as ETM and Spiro-OMeTAD as HTM. The two materials have very different transport properties. In fact, even if the $\mathrm{TiO}_{2}$ is made of sintered nanoparticles, the electron transport can be approximated to charge transport in a crystalline system, with a welldefined conduction band edge and the approximation of parabolic density of states close to the bottom (top) of the conduction (valence) band. On the contrary, the organic material charge transport is based on the transport of polarons and their hopping between adjacent molecules or available energy states. The density of states (DOS) of an organic semiconductor is described by a Gaussian DOS where the center of the Gaussian for the conduction band is levelled to the LUMO (lowest unoccupied molecular orbital) of the molecular/polymeric species and for the valence band to the HOMO (highest occupied molecular orbital):

$$
D(E)=\frac{N_{0}}{\sqrt{2 \pi \sigma^{2}}} \mathrm{e}^{\frac{-\left(E-E_{L, H}\right)^{2}}{2 \sigma^{2}}} .
$$

In eqn (3) $N_{0}$ is the number of energy sites per $\mathrm{cm}^{3}, \sigma^{2}$, the energy variance, is a function of the disorder of the organic material, and $E_{\mathrm{L}(\mathrm{H})}$ is the LUMO (HOMO) energy level. This approximation for organic semiconductors is correct in the non-degenerate case, when the quasi-Fermi levels are within the energy gap and what defines the density are the tails of the Gaussian DOS. The charge density can be calculated from the DOS by assuming a local thermodynamic equilibrium and a quasi-Fermi level and Fermi-Dirac distribution.

\section{Parameterization}

The simulation of the cell requires a careful parameterization of the materials and their electrical properties. Considering the structure, shown in Fig. 1, we need a different parameterization for the HTM, the ETM and the effective material. These parameterizations are not independent, because the effective material is an approximation of the real morphology for the distinct HTM and ETM. In the present work, $\mathrm{TiO}_{2}$ parameters are taken from ref. 48, apart from the recombination constant, 
while those of Spiro-OMeTAD are gathered from literature as reported in Tables 1 and 2. Parameters for the effective material are reported in Table 3.

A specific discussion is required for the parameterization of recombination/generation models and trap density at the interface. These three models are the only ones that change passing from the real morphology where they are related to interface processes, to the effective material where they are related to bulk processes.

Table 1 Parameters for the ETM in the region with the real morphology. All parameters for $\mathrm{TiO}_{2}$ are discussed in ref. 48

\begin{tabular}{llll}
\hline Parameter & Symbol & $\mathrm{TiO}_{2}$ & Units \\
\hline Electron mobility & $\mu_{\mathrm{e}}$ & 0.5 & $\mathrm{~cm}^{2} \mathrm{~V}^{-1} \mathrm{~s}^{-1}$ \\
Hole mobility & $\mu_{\mathrm{h}}$ & 0.5 & $\mathrm{~cm}^{2} \mathrm{~V}^{-1} \mathrm{~s}^{-1}$ \\
Density of states (CB) & $N_{\mathrm{c}}$ & $2.51 \times 10^{19}$ & $\mathrm{~cm}^{-3}$ \\
Density of states (VB) & $N_{\mathrm{v}}$ & $3.25 \times 10^{19}$ & $\mathrm{~cm}^{-3}$ \\
Energy gap & $E_{\mathrm{g}}$ & 4.2 & $\mathrm{eV}$ \\
Relative dielectric const. & $\varepsilon_{\mathrm{r}}$ & 85 & \\
Rec. constant (interface) & $C_{\mathrm{o}}$ & $2 \times 10^{-15}$ & $\mathrm{~cm}^{2} \mathrm{~s}^{-1}$ \\
Trap density (interface) & $N_{\mathrm{t}}$ & $10^{13}$ & $\mathrm{~cm}^{-2}$ \\
Trap dens. exponent & $a$ & 0.3 & \\
& & &
\end{tabular}

Table 2 Parameters for the HTM in the region with the real morphology. For the organic semiconductor the references are directly mentioned in the table

\begin{tabular}{llll}
\hline Parameter & Symbol & Spiro-OM. & Units \\
\hline Electron mobility & $\mu_{\mathrm{e}}$ & 0.001 & $\mathrm{~cm}^{2} \mathrm{~V}^{-1} \mathrm{~s}^{-1}$ \\
Hole mobility & $\mu_{\mathrm{h}}$ & 0.001 (ref. 39) & $\mathrm{cm}^{2} \mathrm{~V}^{-1} \mathrm{~s}^{-1}$ \\
Density of states (CB) & $N_{\mathrm{c}}$ & $10^{19}$ (ref. 38) & $\mathrm{cm}^{-3}$ \\
Density of states (VB) & $N_{\mathrm{v}}$ & $10^{19}$ & $\mathrm{~cm}^{-3}$ \\
Energy disorder (CB) & $\sigma_{\mathrm{e}}$ & 65 (ref. 38) & $\mathrm{meV}$ \\
Energy disorder (VB) & $\sigma_{\mathrm{h}}$ & 65 (ref. 38) & $\mathrm{meV}$ \\
Energy gap & $E_{\mathrm{g}}$ & 2.98 (ref. 42) & $\mathrm{eV}$ \\
Relative dielectric const. & $\varepsilon_{\mathrm{r}}$ & 3.53 & \\
Rec. constant (interface) & $C_{\mathrm{O}}$ & $2 \times 10^{-15}$ & $\mathrm{~cm}^{2} \mathrm{~s}^{-1}$ \\
Trap density (interface) & $N_{\mathrm{t}}$ & - & $\mathrm{cm}^{-2}$ \\
Trap dens. exponent & $a$ & - &
\end{tabular}

Table 3 Parameters for the effective medium. For the discussion of recombination and trap density see the text. For electrons $\mathrm{TiO}_{2}$ parameters are used, while for holes parameters of the HTM from Table 1 are used. The effective energy gap is calculated from the difference between the HOMO of the Spiro-OMeTAD and the conduction band of the $\mathrm{TiO}_{2}$. The dielectric constant of the EM is the Maxwell-Garnett $\mathrm{mix}^{40}$ between the dielectric constants of the two materials, assuming a porosity of 0.5

\begin{tabular}{llll}
\hline Parameter & Symbol & Eff. medium & Units \\
\hline Electron mobility & $\mu_{\mathrm{e}}$ & $0.5 \mathrm{~cm}^{2} \mathrm{~V}^{-1} \mathrm{~s}^{-1}$ & \\
Hole mobility & $\mu_{\mathrm{h}}$ & 0.001 & $\mathrm{~cm}^{2} \mathrm{~V}^{-1} \mathrm{~s}^{-1}$ \\
Density of states (CB) & $N_{\mathrm{c}}$ & $2.51 \times 10^{19}$ & $\mathrm{~cm}^{-3}$ \\
Density of states (VB) & $N_{\mathrm{v}}$ & $10^{19}$ & $\mathrm{~cm}^{-3}$ \\
Energy disorder (VB) & $\sigma_{\mathrm{h}}$ & 65 & $\mathrm{meV}$ \\
Energy gap & $E_{\mathrm{g}}$ & 1.3 (ref. 42 and 43$)$ & $\mathrm{eV}$ \\
Relative dielectric const. & $\varepsilon_{\mathrm{r}}$ & 36.52 & \\
Rec. constant (bulk) & $C$ & $2 \times 10^{-10}$ & $\mathrm{~cm}^{3} \mathrm{~s}^{-1}$ \\
Trap density (bulk) & $N_{\mathrm{t}}$ & $10^{19}$ & $\mathrm{~cm}^{-3}$ \\
Trap dens. exponent & $a$ & 0.3 &
\end{tabular}

Recombination is a fundamental issue for defining the performance of the ss-DSC. For the cell a direct recombination rate of the form:

$$
R=C_{0}\left(n_{\mathrm{e}} n_{\mathrm{h}}-n_{\mathrm{i}}^{2}\right),
$$

is used. In the previous equation $n_{\mathrm{e}}$ and $n_{\mathrm{h}}$ are the electron and hole densities, $n_{\mathrm{i}}$ the equilibrium population in dark conditions and $C_{0}$ a rate constant. Different rate equations have been proposed for standard DSCs. ${ }^{50}$ In the recombination rate, the electron density usually appears with an exponent $\beta$ due to the presence of trap states. ${ }^{49}$ In the present model we have approximated this aspect as it mainly affects the value of the recombination constant $C_{0}$.

The same recombination rate equation is used both in the effective material region and in the real morphology one. The main difference is that in the first case it is a bulk process, in the second it is a surface recombination. This means that even if the recombination equation is the same, the recombination constant cannot be the same, and also because the dimensionality of the constant is not equal in the two cases $\left(\mathrm{cm}^{3} \mathrm{~s}^{-1}\right.$ in the bulk, $\mathrm{cm}^{2} \mathrm{~s}^{-1}$ in the surface case).

Generation is modelled with the Lambert-Beer equation. The thickness of the active region is long enough that standing waves effects will not occur as happens for organic solar cells. The generation is defined as:

$$
G=\int_{\lambda_{1}}^{\lambda_{2}} \alpha(\lambda) \Phi(\lambda) \mathrm{e}^{-\alpha(\lambda) x} \mathrm{~d} \lambda,
$$

where $\Phi$ is the power spectrum of the source (we use a conventional AM 1.5 Sun spectrum), $\alpha$ is the absorption spectrum and $x$ the distance inside the device. The generation is integrated over the entire interval of absorption of the dye, in our case a standard N719 ruthenium dye. Thus, the number of electron-hole pairs photogenerated at every wavelength $G$ is calculated.

Also in this case, as for the recombination term, generation is treated differently passing from the effective material to the real morphology regions. In the real morphology region, we assume a constant generation rate. This approximation can be made considering that the thickness of this region is much smaller compared to the absorption length of N719 (around 0.1-0.2 $\mu \mathrm{m})$. After generation electrons are collected by the ETM and holes by the HTM.

The contacts are modelled as Mott-Schottky contacts, where the injection barriers are kept very small $(0.3 \mathrm{eV}$ between conduction band/HOMO level and contact Fermi energy) and with an efficient injection velocity. We assume that contacts offer a negligible resistance and that the device is limited mainly by bulk recombination.

At the interface between the two materials a density of trap states is also present. ${ }^{27}$ Trap states are modelled with a surface exponential density:

$$
n_{\mathrm{t}}=\int f_{\mathrm{FD}} N_{\mathrm{t}} \mathrm{e}^{\frac{a\left(E-E_{\mathrm{c}}\right)}{k T}} \mathrm{~d} E
$$


with $N_{\mathrm{t}}$ the density of traps, $a$ the trap density exponent, $E_{\mathrm{c}}$ the conduction band edge of the $\mathrm{TiO}_{2}$ and $f_{\mathrm{FD}}$ the Fermi-Dirac distribution of electrons, which depends on the quasi-Fermi energy level.

\section{Merging the effective and the real morphology models}

The concurrent multiscale simulation of the effective and real morphology regions is a delicate issue. The challenge is how to define consistent parameters for the two regions avoiding spurious effects such as charge accumulation or electric fields at the interfaces between the two regions. A simple scheme has been developed to solve this issue: parameters for the real morphology region have been chosen in order to assure that the total recombination and generation in the real morphology region and in an equivalent area using the effective model were the same, i.e.

$$
R_{\mathrm{tot}}=\int_{V_{\mathrm{EM}}} R \mathrm{~d} \Omega \equiv \int_{S_{\mathrm{RM}}} R \mathrm{~d} S,
$$

for recombination and

$$
G_{\mathrm{tot}}=\int_{V_{\mathrm{EM}}} G \mathrm{~d} \Omega \equiv \int_{S_{\mathrm{RM}}} G \mathrm{~d} S
$$

for generation.

In eqn (7) and (8) the first integral is over a volume with the effective medium approximation $\left(V_{\mathrm{EM}}\right)$, while the second along the interface $\left(S_{\mathrm{RM}}\right)$ between ETM and HTM with the real morphology.

The results have been checked by calculating the $J-V \mathrm{~s}$ with and without trap states at the interface using the effective material (EM) or the real morphology (RM) descriptions (see Fig. 2). A small offset is present for $J_{\mathrm{sc}}$ and $V_{\mathrm{oc}}$, the first is more evident without trap states, while the second is particularly present with trap states. The offset in the short circuit current is due to the approximation in the constant

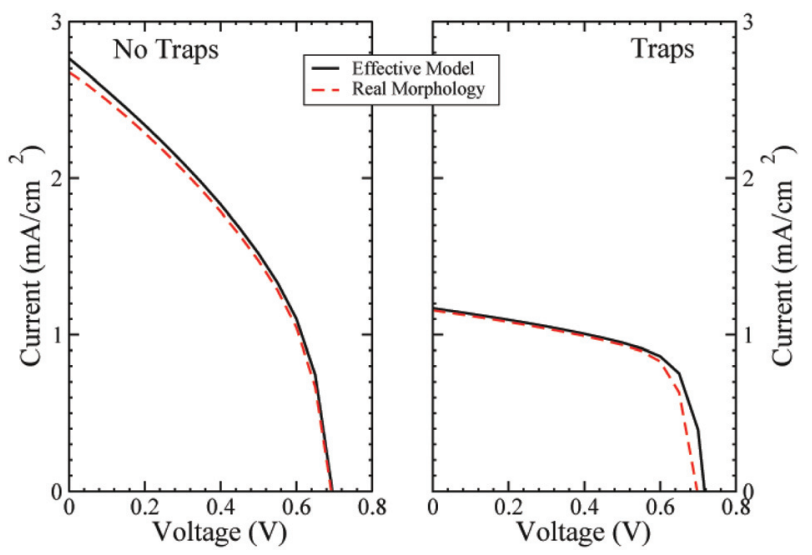

Fig. 2 (Left) $J-V$ characteristics without trap states at the interface with and without real morphology in the middle of the cell. (Right) the same as in the left but adding the trap states at the interface.

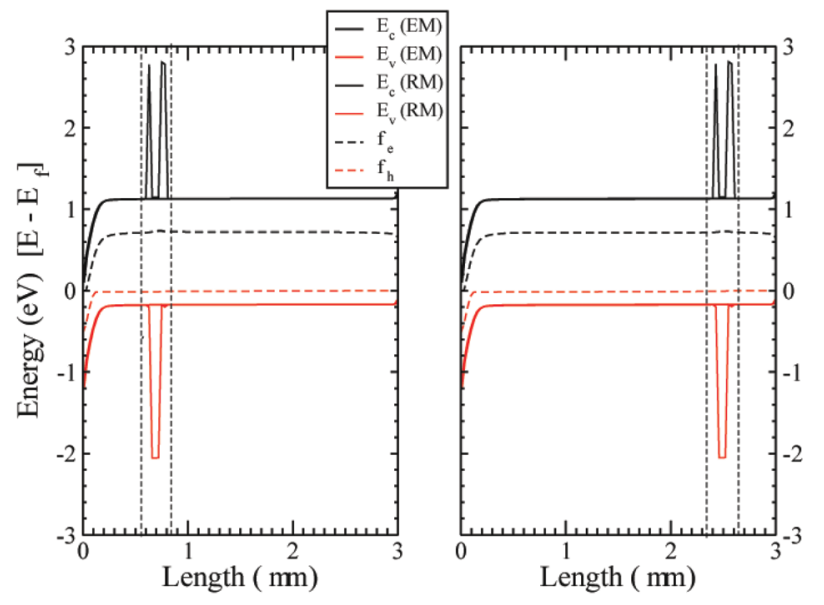

Fig. 3 Conduction and valence bands (LUMO and HOMO level for the HTM) $E_{\mathrm{c}}$ and $E_{\mathrm{v}}$ and electrochemical potentials for electrons $\phi_{\mathrm{e}}$ and holes $\phi_{\mathrm{h}}$ at $J_{\mathrm{sc}}$ for different positions of the RM region. Close to the anode (left) and close to the cathode (right). The band bending is compared to calculations using the effective approximation (EM) only. The $\mathrm{RM}$ region is delimited by the vertical broken lines.

generation model for the RM region, as discussed previously. The difference in open circuit voltage when trap states are considered is induced by the different modelling between RM and $\mathrm{EM}$, as discussed in the next section. The $J-V$ s shown in Fig. 2 present very low efficiency. This is due to the fact that the configuration with traps is a pristine ss-DSC, i.e. a solid state cell where no treatment using the additives has been performed. Also, in the second simulation, with no trap states, we are not considering a fully-functioning ss-DSC, as not all the effects of the additives have been included in the model. Moreover, the dye used as a light absorber, the N719, is not the best for a ssDSC due to the limitations in active layer thickness ( 2 or $3 \mu \mathrm{m})$ in these devices concurrent with the poor molar extinction coefficient of N719. ${ }^{51}$ For this reason other dyes, such as C104 or Z907, are usually used.

As a consistency check to assure that inserting the RM region is not modifying the physics of the device, we have compared the band bending for a pure EM device with that of the device with the RM region added, for different working points and varying the location of the RM region inside the active layer (in the middle, close to the anode or to the cathode). In Fig. 3 the results for the cases close to the anode and the cathode are shown. The conduction and valence bands of the $\mathrm{TiO}_{2}$ and the HOMO and LUMO levels for the organic semiconductor are plotted as well as the quasi-Fermi energy levels, showing both the EM and RM. The agreement between the two models is extremely accurate.

\section{Experimental measurements}

The real geometry of the $\mathrm{TiO}_{2}$ for a portion of a ss-DSC has been reconstructed using an electron tomography dataset with high spatial resolution ${ }^{41}$ (see Fig. 4, top and (a)). In this 


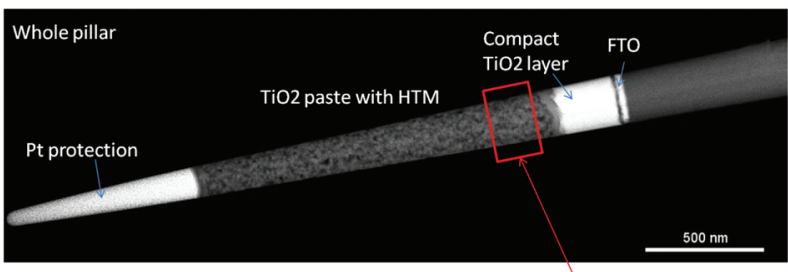

(b)

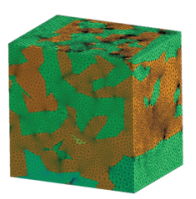

Volume in the reconstruction

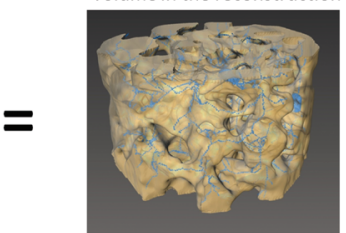

(a) (c)

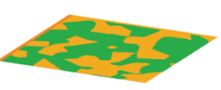

Fig. 4 (Top) High angle annular dark field (HAADF) STEM image. (a) Reconstruction of the $\mathrm{TiO}_{2}$ morphology in a portion of the active layer. (b) Meshing of a cube extracted from the measurement of the $\mathrm{TiO}_{2}$, reconstructing the HTM phase. (c) 2D slice of the 3D morphology.

approach, a transmission electron microscope (TEM) sample is cut out from a ss-DSC using focused ion beam milling (in a FEI Helios Nanolab) employing an annular geometry, resulting in a needle-shaped sample. ${ }^{52,53}$ The needle is lifted out from the device and attached to a TEM-compatible support; further annular thinning is finally employed until the sample is electron-transparent (around 150-200 nm). This choice presents an important advantage compared to the more established approach of extracting a flat lamella: the cylindrical symmetry ensures that the thickness of the sample doesn't increase while tilting, therefore the image quality of the dataset does not degrade for high tilt angles. A tilt series is then acquired in the TEM (FEI Titan operated at $200 \mathrm{kV}$ in STEM mode, tilt range $-76 \rightarrow+76^{\circ}$ ), from which a $3 \mathrm{D}$ reconstruction can be computed. The image was used to process the reconstructed dataset, subtracting the background and applying a threshold for segmentation, isolating the $\mathrm{TiO}_{2}$ volume. Accurate, nanoscale morphological information can thus be retrieved. This allows direct imaging and 3D analysis of both the nanostructured $\mathrm{TiO}_{2}$ network and the HTM component (see Fig. 4).

\section{Effect of traps at the surface of $\mathrm{TiO}_{2}$ mesoporous material on device performance}

The relevance of trapped electrons at the interface between the ETM and HTM is extremely important ${ }^{27}$ and it is one of the reasons for the introduction of ionic additives in the preparation of ss-DSCs. ${ }^{24,25}$ The positive cations of the ionic additives can in fact screen the trapped electrons, changing the electrostatics in the active layer, especially in the HTM due to the lower permittivity of the organic semiconductor compared to the $\mathrm{TiO}_{2}$.

This problem is less severe in standard DSCs, where the liquid electrolyte and high density of ions screen the trapped electrons. The cations accumulate at the interface, screening the electric field within a few ionic layers. ${ }^{54}$

On the contrary, in the case of a ss-DSC, no positive charges are available for the role of screening electrons at the interface, unless directly using the photogenerated holes. This is detrimental for the device operation, considering that the holes contribute to the photocurrent and any perturbation to their distribution could result in a reduction in cell efficiency.

To investigate the effect of trapped electrons we have performed several simulations in the multiscale geometry, including and not including the trap density of states at the interface. These two cases can be considered as limiting cases for an ss-DSC without and with ionic additives, respectively.

We have first investigated the role of trapped charges in an EM approximation (see Fig. 5). The figure shows the free electrons and holes profiles in the cell at $V_{\mathrm{oc}}$. The density of free electrons is reduced in the presence of trap states. The reason for this is that most of the electrons are trapped. The electron quasi-Fermi energy is positioned in the gap and then intercepts the exponential band tail of trap states below the conduction band. Thus, the fraction of trapped electrons is far larger than that of free carriers in the conduction band.

The density of holes is instead increased by the presence of trap states. The negative charge of the trapped electrons attracts holes in the active layer, increasing the equilibrium density. So the overall effect of trap states is to increase hole conductivity and reduce the electron conductivity.

The increase in hole density has a second effect, shown in Fig. 6. The figure presents the conduction, valence and quasiFermi energy levels for a cell with and without trap states. The simulations are performed at $J_{\mathrm{sc}}$ and a RM region is included in the middle of the active layer. In the figure it is clearly shown that the different charge density induced by traps

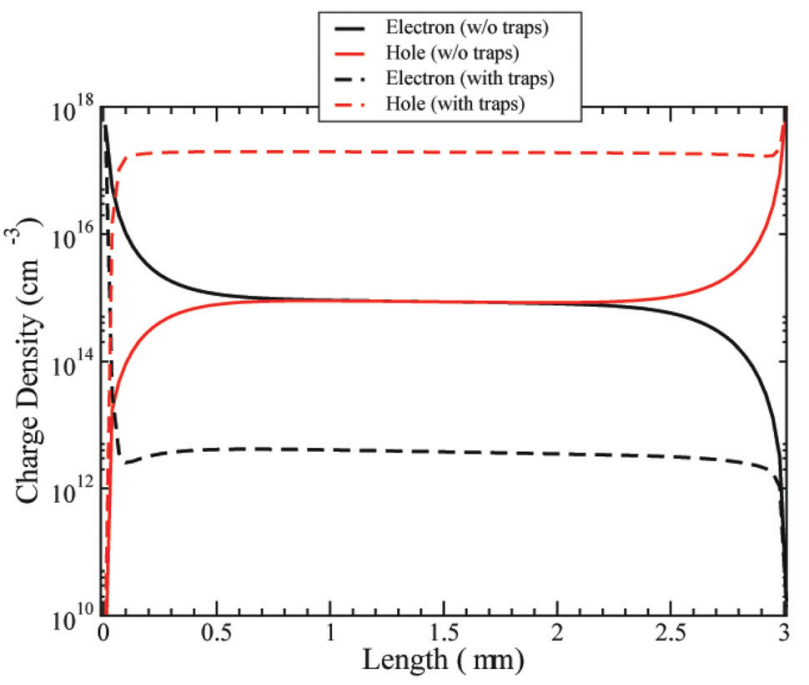

Fig. 5 Density of electrons and holes with and without the presence of trap states at $V_{\text {oc. }}$ Effective model only. 

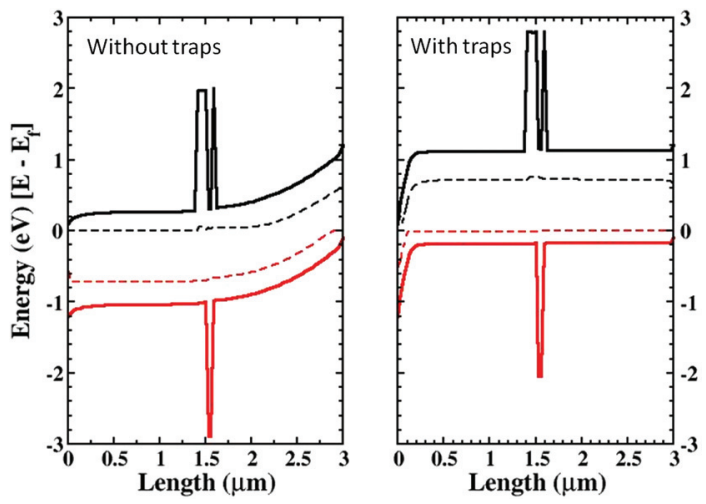

Fig. 6 Effect of band bending at $J_{\mathrm{sc}}$ without (left) and with (right) charged trap states.

changes the band bending in the cell, passing from a pronounced band bending especially close to the cathode (Fig. 6, left) to a flat band situation (Fig. 6, right). The band bending is the signature of a long-range electric field in the active layer. The disappearance of the electric field when trapped electrons are present limits the current (Fig. 2) as the electric field helps charge collection. The presence of a long-range electric field across the active layer in ss-DSCs is under debate. Experimental evidence using impedance spectroscopy points to a purely diffusion-based transport of charge carriers. This could be induced by one of the other effects produced by ionic additives, i.e. the doping of the HTM. However, from our simulations it is clear that the disappearance of a long-range electric field is detrimental to the device operation and that its screening should be considered as a negative effect.

It is known that ionic additives also reduce the interface recombination. ${ }^{23}$ We use the multiscale approach, including the RM in the active layer, to analyze the charge and current distribution in the active layer in more detail. In Fig. 7 the hole and current density at $J_{\mathrm{sc}}$ conditions are shown for the $\mathrm{RM}$ region with and without trapped electrons at the interface. The RM region is inserted close to the cathode contact.

When trapped electrons are present the photogenerated holes accumulate at the interface due to electrostatic attraction. Considering that the recombination at the interface is proportional to the density of electron and holes, a higher density of holes implies a higher recombination. This effect is partially compensated by the lower density of free electrons. Moreover, the current of holes in the presence of traps (Fig. 7e) flows closer to the interface between the two materials. The higher recombination current is particularly evident in the bottleneck of the morphology.

The screening of the local electric field in the RM is shown in Fig. 8. This electric field is particularly intense where trap states are present. Differently from the long range, this local electric field is confined close to the interface and it is more intense in the organic semiconductor due to the lower permittivity.

The analysis of the real morphology can indicate the strengths and weaknesses and the limits of the effective a)

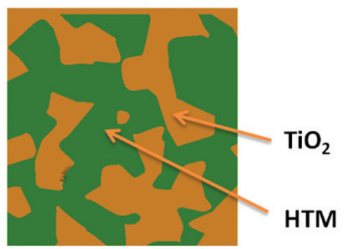

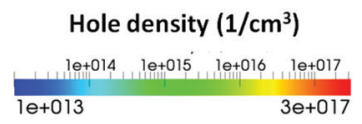

b)

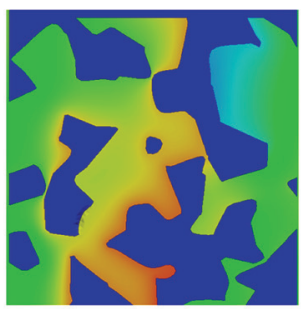

d)

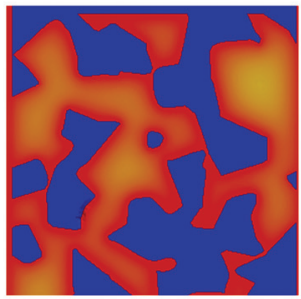

Hole current density $\left(\mathrm{A} / \mathrm{cm}^{2}\right)$

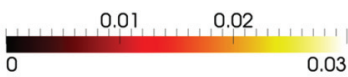

c)

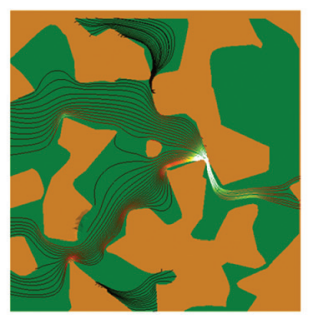

e)

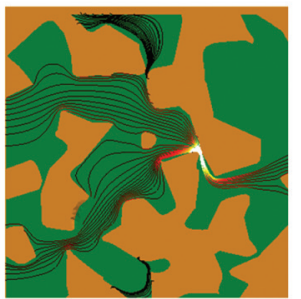

Fig. 7 (a) The RM region with the $\mathrm{TiO}_{2}$ (orange) and the Spiro-OMeTAD (green). (b) Hole density and (c) hole current density at $J_{\text {sc }}$ for the case without trapped electrons at the $\mathrm{TiO}_{2} / \mathrm{HTM}$ interface. (d) and (e) are the same as (b) and (c) but with trapped electrons at the interface.

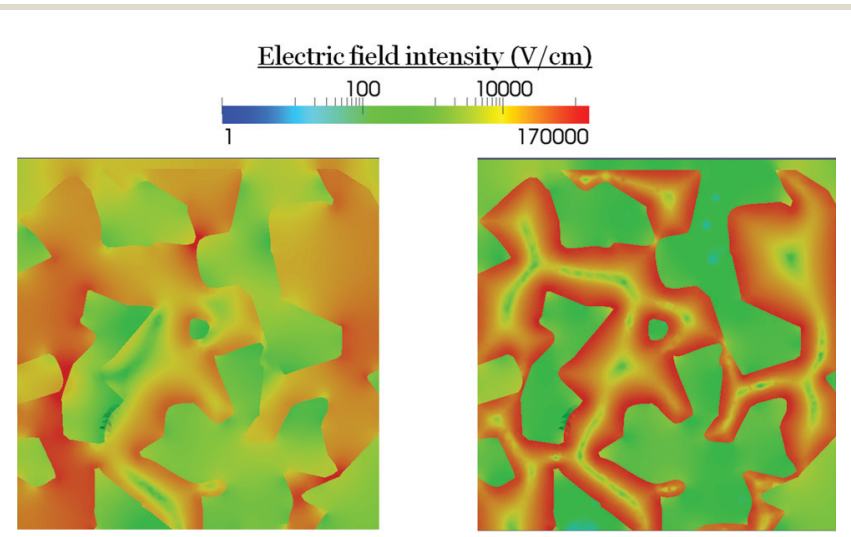

Fig. 8 Electric field distribution inside the RM region at $J_{\mathrm{sc}}$. (Left) without trapped electrons, (right) with trapped electrons.

medium approximation. In particular we have pointed out how the effective medium strongly simplifies the real charge and current distribution inside the two materials, failing in properly describing the electrostatics at the interface. Effects of the morphology, especially if the electron percolation in the $\mathrm{TiO}_{2}$ is difficult, are necessarily lumped into the working parameters in an effective material approximation making their physical interpretation more problematic. 


\section{Conclusion}

In the present work we have analyzed the effect of trap states at the interface in the mesoporous material of a ss-DSC. The electron and hole transport materials were $\mathrm{TiO}_{2}$ and SpiroOMeTAD, respectively. In order to investigate the effect of charged trap states inside the active layer, two different models have been simulated: one using the effective material approximation, the other inserting in a sub region of the cell the real morphology of the mesoporous material. The geometry for the real morphology part was extracted by electron tomography measurements of a real ss-DSC. The insertion of the real morphology region allows us to describe with unprecedented accuracy the electrostatics between electrons and holes and charged trap states at the interface between electron and hole transport materials. The effective material model can in fact only account for the average distribution of charges and electrostatic potential because the real interface is not included.

The simulations show the effect of charged trap states, which induce a higher density of holes in the active layer. The interplay between trapped electrons and holes screens all longrange electric fields, which is detrimental for the cell performance. Introducing a screening mechanism for trapped electrons, the long-range electric field is restored and current increases. The internal electric field in fact helps electrons and holes to reach the correct electrodes, improving the charge collection.

It is known that screening trapped electrons is one of the effects of ionic additives added to the organic semiconductor during the cell preparation. However, experimental measurements using impedance spectroscopy indicate that the transport of photogenerated charges is diffusive, an effect due to the doping of the organic material. Thus, even if the screening of the trapped electrons will restore the long-range electric field, some other effects of the additives prevent its presence. It is anyway clear that the presence of a long-range electric field in the active layer would increase the efficiency of the cell.

Analyzing in more detail the region with the real morphology, another effect becomes evident: the presence of electrons trapped at the interface attracts holes, which accumulate in a thin layer close to the interface. This produces a shift of the hole current close to the interface and enhances recombination. This effect disappears if trapped electrons are not present. A reduction in recombination after the adding of ionic additives has indeed been experimentally observed, and our results point out that the observed electrostatic effect and hole accumulation can be at the base of the experimental recombination modulation with and without ionic additives. These electrostatic effects are particularly important in the hole transport material due to the low permittivity of the organic semiconductor.

The last consideration is about the validity of the effective material model, an approximation widely used for ss-DSCs, but also for other devices like organic photovoltaic cells. Our simulations show that despite the fact that the effective medium approximation can be a valid tool to study devices with complex morphologies, it makes severe approximations in the real distribution of charges and current in the active layer. It just calculates an average value of these quantities. For high performance devices, with no percolation problem in the $\mathrm{TiO}_{2}$, the effective material model is a reasonable approximation of the real system. Its validity decreases rapidly if transport is limited by morphology reasons, in that case an effective material model is forced to include the morphology effects in its transport parameters.

\section{References}

1 Z. Wang, M. Helander, J. Qiu, D. Puzzo, M. Greiner, Z. Hudson, S. Wang, Z. Liu and Z. Lu, Nat. Photonics, 2011, 5, 753-757.

2 D. T. McQuade, A. H. Hegedus and T. M. Swager, J. Am. Chem. Soc., 2000, 122, 12389-12390.

3 M. McCarthy, B. Liu, E. Donoghue, I. Kravchenko, D. Kim, F. So and A. Rinzler, Science, 2011, 332, 570.

4 H. Spanggaard and F. C. Krebs, Sol. Energy Mater. Sol. Cells, 2004, 83, 125-146.

5 U. Bach, D. Lupo, P. Comte, J. E. Moser, F. Weissaertel, J. Salbeck, H. Spreitzer and M. Grätzel, Nature, 1998, 395, 583-585.

6 A. Yella, H. W. Lee, H. N. Tsao, C. Yi, A. K. Chandiran, M. K. Nazeeruddin, E. W. G. Diau, C. Y. Yeh, S. M. Zakeeruddin and M. Grätzel, Science, 2011, 334, 629634.

7 B. O’Regan and M. Grätzel, Nature, 1991, 353, 737-740.

8 R. Sastrawan, J. Beier, U. Belledin, S. Hemming, A. Hinsch, R. Kern, C. Vetter, F. Petrat, A. Prodi-Schwab, P. Lechner and W. Hoffmann, Sol. Energy Mater. Sol. Cells, 2006, 90, 1680-1691.

9 F. Matteocci, G. Mincuzzi, F. Giordano, A. Capasso, E. Artuso, C. Barolo, G. Viscardi, T. M. Brown, A. Reale and A. Di Carlo, Org. Electron., 2013, 14(7), 1882-1890.

10 A. Kojima, K. Teshima, Y. Shirai and T. Miyasaka, J. Am. Chem. Soc., 2009, 131(17), 6050-6051.

11 P. Docampo, J. M. Ball, M. Darwich, G. E. Eperon and H. J. Snaith, Nat. Commun., 2013, 4, 2761.

12 H. J. Snaith and M. Grätzel, Appl. Phys. Lett., 2006, 89, 262114.

13 A. Abate, T. Leijtens, S. Pathak, J. Teuscher, R. Avolio, M. E. Errico, J. Kirkpatrik, J. M. Ball, P. Docampo, I. McPherson and H. J. Snaith, Phys. Chem. Chem. Phys, 2013, 15, 2572-2579.

14 J. Xia, N. Masaki, M. Lira-Cantu, Y. Kim, K. Jiang and S. Yanagida, J. Am. Chem. Soc., 2008, 130, 1258-1263.

15 D. A. R. Barkhouse, H. E. Bishop, B. M. Henry, G. R. Webster, P. L. Burn and H. E. Assender, Org. Electron., 2009, 11, 649-657.

16 D. Bi, F. Wu, W. Yue, Q. Qu, Q. Cui, Z. Qiu, C. Liu, W. Shen and M. Wang, Sol. Energy, 2011, 85, 2819-2825. 
17 A. Abrusci, I. K. Ding, M. Al-Hashimi, T. Segal-Peretz, M. D. McGehee, M. Heeney, G. L. Frey and H. J. Snaith, Energy Environ. Sci., 2011, 4, 3051-3058.

18 U. B. Cappel, A. L. Smeigh, S. Plogmaker, E. M. J. Johansson, H. Rensmo, L. Hammarstrom, A. Hagfeldt and G. Boschloo, J. Phys. Chem. C, 2011, 115, 4345-4358.

19 Q. Wang, Z. Zhang, S. M. Zakeeruddin and M. Grätzel, J. Phys. Chem. C, 2008, 112, 7084.

20 N. Kopidakis, K. D. Benkstein, J. Van de Lagemaat and A. J. Frank, J. Phys. Chem. B, 2003, 107, 11307-11315.

21 P. Tiwana, P. Docampo, M. B. Johnston, L. Herz and H. J. Snaith, Energy Environ. Sci., 2012, 5, 9566.

22 F. Fabregat-Santiago, J. Bisquert, L. Cevey, P. Chen, M. Wang, S. M. Zakeeruddin and M. Grätzel, J. Am. Chem. Soc., 2009, 131, 558-562.

23 J. Kreuger, R. Plass, L. Cevey, M. Piccirelli, M. Gratzel and U. Bach, Appl. Phys. Lett., 2001, 79, 2085.

24 C. A. Kelly, F. Farzad, D. W. Thompson, J. M. Stipkala and G. J. Meyer, Langmuir, 1999, 15, 7047-7054.

25 T. Daeneke, A. J. Mozer, T. H. Kown, N. W. Duffy, A. B. Holmes, U. Bach and L. Spiccia, Energy Environ. Sci., 2012, 5, 7090.

26 I. Mora-Sero and J. Bisquert, Nano Lett., 2003, 3, 945-949.

27 M. C. Fravventura, D. Deligiannis, J. M. Schins, L. D. A. Siebbels and T. J. Savenije, J. Phys. Chem. C, 2013, 117, 8032.

28 D. Kuang, C. Klein, H. J. Snaith, J.-E. Moser, R. HumphryBaker, P. Comte, S. M. Zakeeruddin and M. Grätzel, Nano Lett., 2006, m6, 769.

29 M. Auf der Maur, G. Penazzi, G. Romano, F. Sacconi, A. Pecchia and A. Di Carlo, IEEE Trans. Electron Devices, 2011, 58, 1425.

30 A. Gagliardi, S. Mastroianni, D. Gentilini, F. Giordano, A. Reale, T. Brown and A. Di Carlo, IEEE J. Sel. Top. Quantum Electron., 2010, 16, 1611.

31 A. Gagliardi, D. Gentilini and A. Di Carlo, J. Phys. Chem. C, 2012, 116, 23882-23889.

32 P. R. F. Barnes, A. Y. Anderson, J. R. Durrant and B. C. O'Regan, Phys. Chem. Chem. Phys., 2011, 13, 5798-5816.

33 F. Cappelluti, S. Ma, D. Pugliese, A. Sacco, A. Lamberti, G. Ghione and E. Tresso, Phys. Chem. Chem. Phys., 2013, 15, 14634-14646.

34 J. A. Anta, J. Idgoras, E. Guilln, J. Villanueva-Cab, H. J. Mandujano-Ramrez, G. Oskam, L. Pellej and
E. Palomares, Phys. Chem. Chem. Phys., 2012, 14, 1028510299.

35 J. Villanueva-Cab, G. Oskam and J. A. Anta, Sol. Energy Mater. Sol. Cells, 2010, 94, 45-50.

36 J. Ferber and J. Luther, J. Phys. Chem. B, 2001, 105, 4895-4903.

37 K. Miettunen, J. Halme, A. Visuri and P. Lund, J. Phys. Chem. C, 2011, 115, 7019.

38 J. Garcia-Casidas, F. Fabregat-Santiago, H. J. Bolink, E. Palomares, G. Garcia-Belmonte and J. Bisquert, Synth. Met., 2006, 156, 944-948.

39 A. Abate, D. R. Staff, D. J. Hollmann, H. J. Snaith and A. B. Walker, Phys. Chem. Chem. Phys., 2014, 16, 1132.

40 A. Gagliardi, M. Auf der Maur, D. Gentilini and A. Di Carlo, J. Comput. Electron., 2009, 8, 398-409.

41 P. A. Midgley and R. E. Dunin-Borkowski, Nat. Mater., 2009, 8, 271.

42 T. Leijtens, I. Ding, T. Giovenzana, J. T. Bloking, M. D. McGehee and A. Sellinger, ACS Nano, 2012, 6, 1455-1462.

43 M. Wang, C. Grätzel, S.-J. Moon, R. Humphry-Baker, N. Rossier-Iten, S. M. Zakeeruddin and M. Grätzel, Adv. Funct. Mater., 2009, 19, 2163-2172.

44 A. Gagliardi, M. Auf der Maur and A. Di Carlo, IEEE J. Quantum Electron., 2011, 47, 1214.

45 A. Gagliardi, M. Auf der Maur, D. Gentilini and A. Di Carlo, J. Comput. Electron., 2011, 10, 424.

46 A. Gagliardi and A. Di Carlo, Opt. Quantum Electron., 2012, 44, 141-147.

47 R. Tagliaferro, D. Gentilini, S. Mastroianni, A. Zampetti, A. Gagliardi, T. M. Brown, A. Reale and A. Di Carlo, $R S C$ Adv., 2013, 3, 20273.

48 D. Gentilini, A. Gagliardi, M. Auf der Maur, L. Vesce, D. D'Ercole, T. M. Brown, A. Reale and A. Di Carlo, J. Phys. Chem. C, 2012, 116, 1151-1157.

49 J. Halme, P. Vahermaa, K. Miettunen and P. Lund, Adv. Mater., 2010, 22(35), E210-E234.

50 J. Bisquert, A. Zaban and P. Salvador, J. Phys. Chem. B, 2002, 106(34), 8774-8782.

51 N.-G. Park, J. Phys. Chem. Lett., 2013, 4, 2423.

52 N. Kawase, M. Kato, H. Nishioka and H. Jinnai, Ultramicroscopy, 2007, 107, 8-15.

53 G. Divitini, et al., Adv. Funct. Mater., 2014, 24, 3043-3050.

54 S. Nakade, T. Kanzaki, W. Kubo, T. Kitamura, Y. Wada and S. Yanagida, J. Phys. Chem. B, 2005, 109(8), 3480. 